\title{
Detection of extended-spectrum beta-lactamases in Pseudomonas aeruginosa and Acinetobacter baumannii and their prevalence in Intensive care unit of a tertiary care hospital
}

\author{
Kaur C. ${ }^{1}$, Sharma S. ${ }^{2}$, Sharma P. ${ }^{3}$ \\ ${ }^{1}$ Dr. Charanjeev Kaur, Assistant Professor, ${ }^{2}$ Dr. Sarbjeet Sharma, Professor \& Head, ${ }^{3}$ Dr. Poonam Sharma, Professor; all \\ authors are affiliated with Department of Microbiology, Sri Guru Ram Das Institute of Medical Sciences and Research, \\ Amritsar, Punjab, India.
}

Corresponding Author: Dr. Charanjeev Kaur, Assistant Professor, Department of Microbiology, Sri Guru Ram Das Institute of Medical Sciences and Research, Amritsar, Punjab, India. E-mail: drcharanjeev@gmail.com

\begin{abstract}
Introduction: Pseudomonas aeruginosa and Acinetobacter baumannii have been known to cause variety of infections, among patients admitted in Intensive Care Unit. Non fermenting Gram-negative bacilli are developing resistance to commonly used antibiotics therefore are becoming difficult to treat Among various enzymes produced by bacteria which lead to drug resistance, extended-spectrumbeta-lactamase (ESBL) enzymesis one of the important mechanism of drug resistance. This study that was conducted a) To detect multidrug-resistant $P$. aeruginosa and A. baumannii in patients admitted in ICU patients. b) To determine the prevalence of ESBL producing clinical isolates of Pseudomonas aeruginosa and Acinetobacter sp. in the ICU of the tertiary care hospital. Material and Methods: The study was performed in the microbiology department of a North Indian rural tertiary care hospital (Sri Guru Ram Das Institute of Medical Sciences and Research, Amritsar, India) over a period of one year (January 2012 to December 2012). The study included 100 isolates each of Acinetobacter baumannii \& P. aeruginosa. Identification of both organisms was done using the standard microbiological techniques as described by Colle et al 1996. The antimicrobial susceptibility testing was performed by Kirby Bauer disc diffusion method. To detect ESBL producing isolates phenotypicaly, Disc approximation test was performed. Results: Out of 200 isolates, 100 each of $A$. baumannii and $P$. aeruginosa, weobtained 82 isolates from ICU, $57 \& 25 \mathrm{~A}$. baumannii and $P$. aeruginosa respectively. Among these $57 \mathrm{~A}$. baumannii isolates $89.47 \%$ isolates were resistant to Ceftazidime and among these 33.33\% isolates were ESBL producers.Of 25 P.aeruginosa isolates obtained from ICU $84 \%$ were found to beresistant to ceftazidime by antibiotic sensitivity testing. Among these $44 \%$ were ESBL producers. Conclusion: Our results showed high prevalence ofNon fermenting gram negative bacilli in ICU patient's samples, which were multidrug resistant and producers of Extended spectrum Beta lactamase enzymes.
\end{abstract}

Key words: Acinetobacter baumannii, Disc approximation test, ESBL, ICU, Pseudomonas aeruginosa

\section{Introduction}

Important cause of common infections, occurring in Intensive care units (ICU), like catheter associated urinary tract infections (CAUTI), surgical site infections (SSI), septicemia, are Non fermenting Gram-negative bacilli like Pseudomonas aeruginosa and Acinetobacter baumannii [1]. Non fermenting Gram-negative bacilli are developing resistance to commonly used antibiotics therefore are becoming difficult to treat because genes coding the resistance determinants gets transferred to them from other resistant organismsand also they are intrinsically resistant to some antibiotics. Bacteria become insusceptible to drugs either when they start

Manuscript received: $20^{\text {th }}$ May 2019

Reviewed: $30^{\text {th }}$ May 2019

Author Corrected: $4^{\text {th }}$ June 2019

Accepted for Publication: $8^{\text {th }}$ June 2019 producing enzymes which break down the drugs or target sites of drugs in bacteria are alteredor increased efflux of drugs or decreased permeability of porin channels. Among various enzymes produced by bacteria which lead to drug resistance, extended-spectrumbetalactamase (ESBL) enzymesare one of the important mechanism of drug resistance. ESBL enzymes act on beta lactam drugs like penicillins, early cephalosporins, and monobactams, and render them inactive, but not on cephamycins and carbapenems.

Clavulanic acid, tazobactam and sulbactam are beta lactamase enzyme inhibitors [2]. Genes coding for production of ESBLenzymes are plasmid based. This plasmid is responsible for the spread of ESBLs among

Pathology Update: Tropical Journal of Pathology \& Microbiology Available online at: www.medresearch.in 355 | P a g e 


\section{Original Research Article}

various bacterial strains. Multidrug resistant isolates are a matter of serious concern not only for the physicians while treating their patients but are a serious threat for the hospital infection control committee also. ESBL producing Pseudomonas aeruginosa and Acinetobacter $s p$. have known to cause outbreaks in hospital settings
[3]. Hence, the aim of our study was (a) To identify multidrug- resistant $P$. aeruginosa and $A$. baumannii strains among ICU patients. b) To find the prevalence of Pseudomonas aeruginosa and Acinetobacter sp. isolates which are ESBL producing, in the ICU of the hospital.

\section{Materialand Methods}

The study has been carried out after obtaining the clearance of Institutional ethical committee.

Study design: Prospectivestudy

Study site: Intensive care unit of SGRDIMSR, Amritsar.

Duration of study: January 2012 to December 2012.

Sample size: 100 isolates each of Acinetobacter baumannii \& P. aeruginosa

\section{Inclusion criteria}

Patients admitted in wards during study period were included.

Only Acinetobacter baumannii \& Paeruginosa isolates were included.

\section{Exclusion criteria}

Isolates other than Acinetobacter baumannii \& P.aeruginosa Repeat isolates from same patient.

Sample collection and processing: Clinical samples were collected from patients admitted In various wards, according to standard microbiological guidelines [4]. Identification of both the organisms was done using the standard microbiological techniques as described by Colle et al 1996 [5].

Antibiotic sensitivity testing-The susceptibility of the clinical isolates to some routinely used antibiotics was determined on Mueller Hinton agar by the Kirby-Bauer disk diffusion method using Clinical and Laboratory Standards Institute (CLSI) standards [6]. Antimicrobial agents and their disc concentrations used are as follows;

\begin{tabular}{|c|c|}
\hline Amikacin & $30 \mu \mathrm{g}$ \\
\hline Ciprofloxacin & $5 \mu \mathrm{g}$ \\
\hline Ceftazidime & $30 \mu \mathrm{g}$ \\
\hline Piperacillin-tazobactam & $100 / 10 \mu \mathrm{g}$ \\
\hline Imipenam & $10 \mu \mathrm{g}$ \\
\hline Meropenem & $10 \mu \mathrm{g}$ \\
\hline Polymxin B & $300 \mathrm{units}$ \\
\hline Chloramphenicol & $5 \mu \mathrm{g}$ \\
\hline Gentamicin & $10 \mu \mathrm{g}$ \\
\hline Norfloxacin & $10 \mu \mathrm{g}$ \\
\hline
\end{tabular}

The discs were obtained from Hi Media laboratories Pvt Limited

The results were interpreted as per CLSI (CLINICAL LABORATORY STANDARDS INSTITUTE)[6]. E.coli ATCC 25922 was used ascontrol organism for antibiotic sensitivity.

Disc approximation method [6]- Isolates found resistant or with decreased susceptibility to Ceftazidime (30 $\mu \mathrm{g})$ third generation cephalosporin antibiotics were subjected to Disc approximation method, a phenotypic test for detection of ESBL production. A disc of Ceftazidime - clavulanic acidand second disc containing Ceftazidime aloneis placed on Mueller hinton agar plate which is inoculated with the test strain, at a distance of $15 \mathrm{~mm}$ from each other. If zone of 


\section{Original Research Article}

inhibition around ceftazidime- clavulanic aciddiscis $\geq 5$ mmlarger than that around the ceftazidime disc alone was interpreted as confirmatory for ESBL production as per Clinical and Laboratory Standards Institute (CLSI) 201 guidelines [6]. When performing the ESBL confirmatory tests, K. pneumoniae ATCC 700603 and E. coli ATCC 25922 was tested routinely. E. coli ATCC 25922: $\leq 2-\mathrm{mm}$ increase in zone diameter for antimicrobial agent tested alone vs its zone when tested in combination with clavulanic acid was taken as negative control. K. pneumoniae ATCC 700603: $\geq 5$ $\mathrm{mm}$ increase in ceftazidime clavulanic acid zone diameter was taken as positive control to standardize the test [6].

Test for ESBL Production

\section{Result}

Out of total 200 isolates, 100 each of $A$. baumannii and $P$. aeruginosa, 82 isolates were obtained from ICU. Among these 82 isolates, 57 were $A$. baumannii and 25 isolates were $P$. Aeruginosa (Table no:1) followed by 63 isolates from surgery ward $18 \& 45$ isolates each of $A$. baumannii and $P$. aeruginosa respectively while 16 from medicine ward 6 and 10 each of $A$. baumannii and $P$. aeruginosa respectively.

Table No-1: Distribution of A.baumannii \& P. aeruginosa isolates in various wards of institute

\begin{tabular}{|c|c|c|c|}
\hline & A. baumannii & P. aeruginosa & Total \\
\hline ICU & 57 & 25 & 12 \\
\hline Emergency ward & 7 & 5 & 2 \\
\hline Eye ward & 0 & 2 & 5 \\
\hline Gynae ward & 4 & 10 & 16 \\
\hline Medicine ward & 6 & 9 & 8 \\
\hline Ortho ward & 3 & 3 & 63 \\
\hline Paed ward & 5 & 45 & $\mathbf{2 0 0}$ \\
\hline Surgery ward & 18 & $\mathbf{1 0 0}$ & 12 \\
\hline Total & $\mathbf{1 0 0}$ & 10 & 8 \\
\hline
\end{tabular}

Table no 2 shows maximum $A$. baumannii isolates resistant to ceftazidime $87 \%$, followed by amino glycosides (Gentamicin 80\%, Amikacin 72\%), quinolones (Norfloxacin 84.2\%, Ciprofloxacin 70\%). Susceptibility ofthe isolates was maximum (100\%) to Polymyxin B.

Table No-2: Antibiotic Susceptibility Pattern of $A$. baumannii \& $P$. aeruginosaisolated from various wards

\begin{tabular}{|l|c|c|c|c|c|c|}
\hline \multirow{2}{*}{ Antimicrobials } & \multicolumn{3}{|c|}{ A. baumanni } & \multicolumn{3}{c|}{ P. aeruginosa } \\
\cline { 2 - 8 } & Sensitive & Intermediate & Resistant & Sensitive & Intermediate & Resistant \\
\hline Amikacin & 26 & 2 & 72 & 91 & 3 & 6 \\
\hline Gentamicin & 19 & 1 & 80 & 52 & 0 & 48 \\
\hline Ciprofloxacin & 26 & 4 & 70 & 41 & 0 & 59 \\
\hline Ceftazidime & 13 & & 87 & 38 & 1 & 62 \\
\hline Pipracillin-tazobactum & 47 & 0 & 53 & 82 & 0 & 17 \\
\hline Imipenem & 66 & 2 & 32 & 75 & 0 & 25 \\
\hline Meropenem & 42 & 0 & 58 & 64 & 0 & 36 \\
\hline Chloramphenicol & 17 & 0 & 64 & 13 & 0 & 69 \\
\hline Norfloxacin & 3 & 0 & 16 & 7 & 0 & 11 \\
\hline Polymixin-B & 100 & 0 & 0 & 100 & 0 & 0 \\
\hline
\end{tabular}

As shown in table no 2 maximum $P$. aeruginosaisolates were resistant to chloramphenicol $69 \%$, followed by ceftazidime $62 \%$, quinolones (norfloxacin 61\%, ciprofloxacin 59\%), aminoglycosides (gentamicin 48\%, amikacin 6\%), meropenem $36 \%$. No isolate was found to be resistant to Polymyxin B. 
Original Research Article

Table No-3: Antibiotic Susceptibility Pattern of $A$. baumannii \& P. aeruginosaisolated from ICU

\begin{tabular}{|l|c|c|c|c|c|c|}
\hline \multicolumn{1}{|c|}{ Antimicrobials } & \multicolumn{3}{|c|}{ A. baumannii } & \multicolumn{3}{c|}{ P. aeruginosa } \\
\hline & Sensitive & Intermediate & Resistant & Sensitive & Intermediate & Resistant \\
\hline Amikacin & 12 & 2 & 43 & 12 & 2 & 11 \\
\hline Gentamicin & 12 & 0 & 45 & 07 & 0 & 18 \\
\hline Ciprofloxacin & 16 & 3 & 38 & 05 & 0 & 20 \\
\hline Ceftazidime & 06 & 0 & 51 & 04 & 0 & 21 \\
\hline $\begin{array}{l}\text { Pipracillin- } \\
\text { tazobactum }\end{array}$ & 24 & 0 & 33 & 18 & 1 & 6 \\
\hline Imipenem & 35 & 2 & 20 & 15 & 0 & 10 \\
\hline Meropenem & 23 & 0 & 34 & 09 & 0 & 16 \\
\hline Chloramphenicol & 12 & 0 & 44 & 05 & 0 & 20 \\
\hline Polymixin-B & 57 & 0 & 0 & 25 & 0 & 0 \\
\hline
\end{tabular}

Distribution of Ceftazidime resistant $A$.baumannii isolates isolated from various specimens in ICU is shown in table 4. Maximum number of isolates $45.61 \%$ (26) were obtained from endotracheal tube (ETT) secretions from ICU patients, of which $96.15 \%$ (25) were resistant to Ceftazidime, followed by $26.3 \%(15)$ isolates from pus out of which $86.66 \%$ (13) isolates were not susceptible to Ceftazidime.

Table No-4: Distribution of various samples from ICU from which $A$. baumannii was isolated

\begin{tabular}{|c|c|c|c|}
\hline Sample & A. baumannii from ICU & $\begin{array}{c}\text { CEFTAZIDIME } \\
\text { RESISTANT }\end{array}$ & ESBL PRODUCER \\
\hline $\begin{array}{c}\text { Endotracheal tube } \\
\text { Secretion }\end{array}$ & 26 & $25(96.15 \%)$ & $6(23.07 \%)$ \\
\hline Pus & 15 & $13(86.66 \%)$ & $6(40 \%)$ \\
\hline Blood & 10 & $8(80 \%)$ & $3(30 \%)$ \\
\hline Sputum & 3 & $3(100 \%)$ & $3(100 \%)$ \\
\hline Body Fluids & 2 & $1(50 \%)$ & $1(100 \%)$ \\
\hline Urine & 1 & $1(100 \%)$ & $\mathbf{1 9}(\mathbf{3 3 . 3 3 \% )}$ \\
\hline Total & $\mathbf{5 7}$ & $\mathbf{5 1 ( 8 9 . 4 7 \% )}$ & 0 \\
\hline
\end{tabular}

Out of 26 isolates obtained from ETT Secretions, 6 isolates were to be ESBL producers while 6 isolates from pus were ESBL producers (Table no 4)

In our study number of P.aeruginosaisolatesisolatedfrom ICU were 25. (Table No 1) There antibiotic sensitivity test showed $84 \%$ (21) were resistant to ceftazidime (Table no 3).

These ceftazidime resistant isolates when subjected to Disc approximation test showed 44\% (11) isolates were ESBL producers (Table no 5)

Table No-5: Distribution of various samples from ICU from whichP.aeruginosawas isolated.

\begin{tabular}{|c|c|c|c|}
\hline Sample & P.aeruginosa from ICU & Ceftazidime Resistant & ESBL Producer \\
\hline Endotracheal tube Secretion & 11 & $10(90.9 \%)$ & $5(45.45 \%)$ \\
\hline Pus & 7 & $7(100 \%)$ & $4(57.14 \%)$ \\
\hline Urine & 5 & $3(60 \%)$ & $2(40 \%)$ \\
\hline Blood & 1 & $1(100 \%)$ & 0 \\
\hline Body fluids & 1 & 0 & 0 \\
\hline Sputum & 0 & 0 & $\mathbf{1 1 ( 4 4 \% )}$ \\
\hline Total & $\mathbf{2 5}$ & $\mathbf{2 1 ( 8 4 \% )}$ & 0 \\
\hline
\end{tabular}




\section{Original Research Article}

Maximum number of $P$. aeruginosa isolates 44\% (11) were obtained from endotracheal tube secretions in ICU (Table-5), out of which $90.9 \%$ (10) were not susceptible to Ceftazidime, followed by $28 \%$ (7) isolates obtained from pus samples of ICU patients and all the 7 isolates were resistant to ceftazidime.

Disc approximation test shows that $45.45 \%$ (5) isolates from ETT secretions and $57.14 \%$ (4) isolates from pus were ESBL producers. (Table no5)

\section{Discussion}

Beta-lactamases are classified into fourclasses, to be precise $\mathrm{A}, \mathrm{B}, \mathrm{C}$, and $\mathrm{D}$, which is based on the amino acids sequence homology. According to Ambler classification A, C, and D classes are called serine-betalactamases, and $\mathrm{B}$ class beta-lactamases are referred to as MBL [7]. ESBLs are plasmid mediated $\beta$-lactamases that confer resistance to broad spectrum $\beta$ - lactum antibiotics including third and fourth generation cepahlosporins, azetronam, and extended spectrum penicillins.

These plasmids often encode mutations which confere resistance to other broad spectrum agents including aminoglycosides, co-trimoxazole and fluoroquinolones, resulting in organism resistant to most broad spectrum antibiotics [8].

In the present study $87 \%$ A.baumannii were resistant to ceftazidime, while $72 \%, 70 \%, 58 \%$ were resistant to Amikacin, Ciprofloxacin, Meropenem respectively. Results were slightly different in the study by Karthik et al $89 \%, 80 \%, 72 \%$ of $A$. baumannii isolates were resistant to Meropenem, Amikacin \& Ciprofloxacin respectively andresistance to Ceftazidime was $(36 \%)$ [9]. In another study all Acinetobacter isolates were $100 \%$ resistant to all generations of Cephalosporins \& Trimethoprim/ sufamethoxazole and $80-90 \%$ resistant to aminoglycosides and beta lactam/ beta lactamase inhibitor combination [10], these are the drugs which are commonlybeing prescribed in the hospital.

However, lesser used antimicrobials like polymyxin B were $100 \%$ sensitive. Such observations have also been observed by other investigators wherein susceptibility is attributed to decreased usage of those antimicrobials [11].

Our study revealed (62\%) P.aeruginosaisolates to be resistant to Ceftazidime while in a previous study by Aggarwal et al resistance to Ceftazidime was $10.35 \%$. Among aminogly cosides, Amikacin showed least resistance, $6 \%$ in our study. Similar results were shown in the study by Aggarwal et al [12] While strainsresistant to Gentamicin were $48 \%$ in our study, same results were shown by Sarkaret al (45\%). We observed (59\%) resistance to Ciprofloxacin while in the study of Sarkaret alit was slightly lower i.e (50\%)[13].
All the isolates were $100 \%$ sensitive to Polymyxin B , same results were also recordedby Aggarwal et al\& Sarkar et al[12][13] .

Our study reported very high incidence of ESBL among P.aeruginosa from ICU(44\%) similar results were observed by Goel et al[14]. Butresults by Agarwal et al were different which showed $20.27 \%$ of ESBL production [15]. Typical ESBL production was observed in $33.33 \%$ among A.baumanniiin the present study while study by Goel et alshows 17.95\% prevalence of ESBL and in other studies, ESBL production has been found to range from 20 percent in India to $54.6 \%$ in Korea [16].

Another study revealed that 24.3 per cent of NFGNB isolated from ICU patients were ESBLproducers [10]. In this study, Amikacin, Polymyxin B demonstrated maximum sensitivity against NFGNB. Therefore, use of these antibiotics should be restricted to severe infections, especially in critically ill ICU patients, to avoid rapid emergence of resistant strains.

In our study, ESBL producing $P$. aeruginosa and $A$. baumannii were frequently isolated from endotracheal secretions ( $45.45 \%$ and $23.07 \%$, respectively), as also shown by Abd El-Fattah [17].

\section{Conclusion}

It can be concluded from the study that A.baumannii and P.aeruginosa are emerging as leading hospital pathogens. Resistance to commonly used antibiotics is posing therapeutic challenge to the clinicians. Such strains have been implicated in many recent out breaks mostly in ICUs where extensive use of antibiotics has contributed to the selection of highly resistant strains. The organisms are resistant due to various factors, especially production of ESBL anddetecting them will help in the study of epidemiology of these organisms and hospital infection control programme.

They can obtain resistance determinants and can exist inhospital environment for prolonged period. By performing a simple, easy and economical test i.e disc potentiation test ESBL producing organisms can be diagnosed 


\section{Original Research Article}

A major problem with ESBLs is their capacity to cause therapeutic failure with cephalosporins and azetreonam when host organism appears to be susceptible to these agents in laboratory tests.

Hence, CLSI recommends that laboratories should report ESBL producing isolates as resistant to all penicillins, cephalosporins (including cefepime and cefpirome), and azetreonam irrespective of in-vitro test results.

The carbapenems (Ertapenem, Meropenem and Imipenem) are currently considered the drug of choice for serious infections caused by these pathogens. Piperacillin-Tazobactam and Cefoperazone- Sulbactam may be considered options in mild infections and when ESBL producers are demonstrably susceptible in -vitro.

Moreover, it is important to implement antibiotic restriction policies to avoid excessive and injudicious use of extended spectrum cephalosporins and Carbapenems in every hospital. Depending upon the antimicrobial resistance testing, antibiotic policy of the hospital isprepared. Drugs like Carbapenems, Polymyxinsare kept as reserve drugs.

Findings: Nil; Conflict of Interest: None initiated Permission from IRB: Yes

\section{References}

1. Malini A, Deepa E, Gokul B, et al. Nonfermenting gram-negative bacilli infections in a tertiary care hospital in kolar, karnataka. J Lab Physicians. 2009 Jul; 1(2):62-6. DOI:10.4103/0974-2727.59701

2. Gupta V. An update on newer beta-lactamases. Indian J Med Res. 2007 Nov;126(5):417-27.

3. AgarawalP, Gosh AN, Kumar S, Basu B, Kapila K. Prevalence of extended spectrum beta lactamases among E.coli and Klebsiella pneumoniae isolates in a tertiary care hospital. IJPM 2008; 51(1):139-142. DOI:10. 4103/ 0377-4929.40428

4. J.G Colle and W. Marr. Specimen collection, culture containers and media," in Mackie and McCartney Practical Medical Microbiology, G.J Collee, G.A. Fraser, B.P Marmon and A. Simmons, Eds., pp.14-27, Elseviar, Toronto, Canada,14 edition, 2008.

5. J.G Colle ,R.S Miles and B.Watt. Test for identification of bacteria," in Mackie and McCartney Practical Medical Microbiology, G.J Collee, G.A. Fraser, B.P Marmon and A. Simmons, Eds., pp.131150, Elseviar, Toronto, Canada, 14 edition, 2008.
6. Clinical Laboratory Standard Institute. Performance Standards for Antimicrobial Susceptibility Testing; Twenty-Second Informational Supplement. Vol. 32. Clinical Laboratory Standard Institute; Wayne, Pennsylvania, USA: 2012. pp. 70-71.

7. Koneman EW, Allen SD, Jand WM, Schreckenberg PC. Colour Atlas and Text Book of Diagnostic Microbiology. 6th ed. San Francisco: Lippincott;2006. p. 955-63.

8. National treatment guidelines for Antimicrobial use in Infectious Diseases, version 1.0 National Centre for Disease Control; 2016;42

9. Uma Karthika R, Srinivasa Rao R, Sahoo S, et al. Phenotypic and genotypic assays for detecting the prevalence of metallo-beta-lactamases in clinical isolates of Acinetobacter baumannii from a South Indian tertiary care hospital. J Med Microbiol. 2009 Apr; 58 (Pt 4):430-5. doi: 10.1099/jmm.0.002105-0.

10. Goel. N, Punia. P, Chaudhary U. Prevalence of ESBL, MBL and Amp C producing XDR Acinetobacter isolates from lower respiratory tract specimens. International Journal of Contemporary Medical Research 2017;4(10):2091-2095.

11. Manikal VM, Landman D, Saurina G, et al. Endemic carbapenem-resistant Acinetobacter species in Brooklyn, New York: citywide prevalence, inter institutional spread, and relation to antibiotic usage. Clin Infect Dis. 2000 Jul;31(1):101-6. Epub $2000 \mathrm{Jul}$ 17. DOI: $10.1086 / 313902$

12. Agrawal G, Lodhi R B, Kamalakar U P, Khadse R K, Jalgaonkar S V, Study of Metallobeta lactamase production in clinical isolates of Pseudomonas aeruginosa, IJMM 2008; 26 (4): 349-51.DOI:10.4103/ 0255- 0857. 43573 PMID: 18974488

13. Sarkar B, Biswas D, Prasad R, et al. A clinicomicrobiological study on the importance of pseudomonas in nosocomially infected ICU patients, with special reference to metallo beta1-lactamase production. Indian J Pathol Microbiol. 2006 Jan;49 (1): 44-8.

14. Goel V, Hogade SA, Karadesai SG. Prevalence of extended-spectrum beta-lactamases, Amp $\mathrm{C}$ betalactamase, and metallo-beta-lactamase producing Pseudomonas aeruginosa and Acinetobacter baumannii in an intensive care unit in a tertiary Care Hospital. J Sci Soc 2013; 40(1): 28-31. DOI:10.4103/0974-5009. 109691. 
15. Aggarwal R, Chaudhary U, Bala K. Detection of extended-spectrum beta-lactamase in Pseudomonas aeruginosa. Indian J Pathol Microbiol 2008;51(2): 222-4. DOI:10.4103/0377-4929.41693.

16. Joshi SG, Litake GM, Ghole VS, et al. Plasmidborne extended-spectrum beta- lactamase in a clinical isolate of Acinetobacter baumannii. J Med

\section{Original Research Article}

Microbiol. 2003 Dec; 52 (Pt 12):1125-7. DOI:10.1099/ 0022-1317-52-12-1125

17. Abd El-Fattah SM. Evaluation of antibiotic resistance among Gram-ve bacilli isolated from critically ill patients: Relation to risk factors and liberal use of antibiotics.M.Sc Thesis in Medical Microbiology and Immunology. Faculty of Medicine. Giza, Egypt: Cairo University; 2008.

\section{How to cite this article?}

Kaur C, Sharma S, Sharma P. Detection of extended-spectrum beta-lactamases in Pseudomonas aeruginosa and Acinetobacter baumannii and their prevalence in Intensive care unit of a tertiary care hospital. Trop J Path Micro 2019; 5(6):355-361.doi:10.17511/jopm.2019.i06.04. 